\title{
A Novel Device for the Calibration of Sonic and Ultrasonic Recording Transducers
}

\author{
Matthew S. McMurray ${ }^{\mathrm{a}}$ and Devin K. Hubbard ${ }^{\mathrm{b}}$ \\ aDepartment of Psychology, University of Illinois at Chicago, 1007 W. Harrison St. M/C 285, \\ Chicago, IL, 60607 USA, +1-312-996-5370, mcmurray@uic.edu \\ bDepartment of Biomedical Engineering, University of North Carolina, 401 Taylor Hall CB\# 7575, \\ 109 Mason Farm Rd., Chapel Hill, NC, 27599, +1-919-843-6076, dhubbard@email.unc.edu
}

\section{Abstract}

Recently, there has been an increase in the analysis of animal vocalizations in behavioral neuroscience as a social cue or indicator of neurological integrity. Despite the multitude of researchers examining vocalizations in a variety of species, no inexpensive, tunable devices currently exist to calibrate the amplification applied to such vocalizations before data are collected. Many commercially available recording systems have analogue adjustments for gain, but such methods are notoriously unreliable and highly variable. Without a consistent level of gain, the amplitudes of recorded acoustic signals cannot be reliably compared. Here, we describe an apparatus designed to fulfill this need, which we have labeled the Calibration Unit for Recording Transducers (CURT). To maximize application to various fields, its emitted frequency and amplitude are tunable to output frequencies in both human-sonic $(20 \mathrm{~Hz}-20 \mathrm{kHz})$ and human-ultrasonic ranges $(20-100 \mathrm{kHz})$. Additionally, it is a portable (weighing approximately 180 $\mathrm{g}$ ), customizable, stand-alone unit, and fits a variety of microphone connector types. The CURT is also relatively low cost to build (under 250.00 USD), thereby making such a device available to as many researchers as possible in animal behavior and neuroscience.

\section{Keywords}

Acoustic; Calibration; Vocalization; Ultrasonic; Frequency; Amplitude

\section{Introduction}

The study of animal vocalizations in behavioral and neurobiological research has become increasingly popular over the last decade. Prior to the year 2000, the term "vocalization" was found by Pubmed in only 3979 papers. Between then and the year 2012, this number has effectively doubled, with over 4100 additional papers added. This increase in interest by the scientific community may be the result of numerous factors, but perhaps specifically due to the relevance of vocalizations in countless species as both a social cue and an important biological signal (Zeskind et al., 2011). Given its translational nature and our general

(C) 2013 Elsevier B.V. All rights reserved.

Corresponding Author: Matthew S. McMurray, 1007 W. Harrison St. M/C 285, Department of Psychology, Chicago, IL, 60607 USA, 312-996-5370, mcmurray@uic.edu.

Publisher's Disclaimer: This is a PDF file of an unedited manuscript that has been accepted for publication. As a service to our customers we are providing this early version of the manuscript. The manuscript will undergo copyediting, typesetting, and review of the resulting proof before it is published in its final citable form. Please note that during the production process errors may be discovered which could affect the content, and all legal disclaimers that apply to the journal pertain. 
understanding of the physiological mechanisms of cry production, one can assume that the popularity of vocalizations will continue to increase. However, further adoption of this endpoint must be tempered by the development of accurate tools to assess the various spectral parameters, including frequency, amplitude, harmonics, duration, repetition rate, and waveform complexity (shape), and melody.

The accurate quantification of spectral characteristics in any species requires accurate recording, amplification, digitization, and analysis. For many labs, this can be accomplished via commercially available systems, such as the popular Computerized Speech Lab (KayPentax, Montvale, NJ) used in human speech research, or the UltraSoundGate (Avisoft Bioacoustics, Berlin) used in rodent, birdsong, and bat research. These are turn-key systems that provide ease of utility, but at the cost of reduced flexibility and/or specialization. Other labs, including our own, have resorted to custom-built solutions, thereby granting flexibility or specialization, but at the cost of increased system development and training costs. No matter the tool selected, it is important to understand its limitations and constraints at each level of acquisition. For example, sampling rates in analogue to digital converters should be taken into consideration. Sampling at too high of a rate will cause aliasing artifacts and sampling at too low of a rate will prevent accurate reconstruction of the entire waveform. To determine the appropriate sampling rate, a popular heuristic is the Nyquist-Shannon Theorem, (Nyquist, 1928; Shannon, 1949) which states that one must record at a minimum of twice the input signal frequency (i.e. one must sample a $2 \mathrm{~Hz}$ signal at a minimum of 4 $\mathrm{Hz}$ to be able to capture the signal correctly); however, many consider the Nyquist frequency to be the minimum acceptable sampling rate. Similarly, when recording ultrasonic vocalizations $(>20 \mathrm{kHz})$, common to rodent and bat species, using a microphone with a frequency response range lower than $20 \mathrm{kHz}$ would not be appropriate, since sampling sounds outside the frequency response range of a microphone results in dramatic attenuation of the signal due to non-linear frequency response of microphones.

The primary function of a microphone is to convert the physical movement of air into a change in electrical potential (voltage) that can be measured or recorded. This conversion is accomplished via a transducer and results in minute changes in voltage ( $\mathrm{mV}$ range). In our equipment, there is an exponential relationship between voltage and amplitude (dB, see Figure 1), with the range and shape dependent upon the specific hardware used. Such small changes in signal typically require amplification before they can be effectively digitized, especially when sounds occur at low volumes. Amplification (i.e. recording volume adjustment in audio systems) is one often overlooked limitation of many recording systems. Variations in amplification can result in inaccurate quantification of a sound's amplitude, thus biasing measurements of the sound duration (due to clipping) and frequency. On most commercial systems, amplification (gain) is accomplished via an analogue "volume knob". In some commercial systems, this knob has numeric feedback to inform the user of the approximate level of amplification, but more often than not there is no objective feedback available. Without accurate feedback of the level of amplification, it is likely that amplification levels will vary between recordings, potentially confounding results. Additionally, when using multiple microphones/amplifiers, it is critical to ensure each system is representing amplitude in the same manner. Thus, calibration of amplification systems is of paramount importance to ensure measurement is as accurate as possible, especially when the level amplification is set via an analogue mechanism.

Despite the multitude of researchers currently examining vocalizations, no calibration device has been widely adopted for use in the field. The two commercial recording systems mentioned above (Computerized Speech Lab and UltraSoundGate) both have analogue adjustments for gain, but only Avisoft (manufacturer of UltraSoundGate) supplies an inexpensive calibration device (Model 60105), and this device has a fixed frequency output 
$(40 \mathrm{kHz})$, limiting its usage in other fields. Other calibration devices, such as the G.R.A.S. Sound and Vibration, Sound Calibrator Type $42 \mathrm{AB}$, can produce a calibration tone in a variety of frequencies, but are limited in range $(<10 \mathrm{kHz})$. Given the increasing adoption of vocalization measures, a need exists to develop a calibration tool for a variety of frequency and amplitude ranges. Here we describe an apparatus, which we have labeled the Calibration Unit for Recording Transducers (CURT), designed to fulfill the needs described. The CURT was designed to maximize utility in a number of fields, and can be easily customized to suit the specific needs of most labs. The emitted frequency and amplitude are tunable to output frequencies in both human-sonic $(20 \mathrm{~Hz}-20 \mathrm{kHz})$ and human-ultrasonic ranges (20-100 $\mathrm{kHz}$ ), up to a maximum frequency of approximately $100 \mathrm{kHz}$. Additionally, it is designed to have the capability of being a highly portable, stand-alone unit that fits a variety of microphone connector types. The CURT is also very low cost to build (under 250.00 USD), thus enabling adoption by many researchers.

\section{Circuit Design}

The CURT is composed of two main portions: the internal driving circuitry (enclosed within a custom made ABS and acrylic enclosure), and the external calibration source (enclosed in a custom mounting bracket tailored specifically to the transducer to be calibrated). The entire apparatus weights $180 \mathrm{~g}$, and the driving electronics fit into a box that is $107 \mathrm{~mm} \times 70 \mathrm{~mm} \times 56 \mathrm{~mm}$.

The basic flow-diagram of the circuit is provided in Figure 2 (circuit diagram provided in Supplementary Material $\dagger$ ). The microprocessor used was a PIC18F4550 (Microchip Technology Inc., Chandler, AZ). This chip was selected to provide the basic functionality required of the calibration unit, while providing the possibility of USB connectivity in the future. The circuit derives its clock from an external crystal oscillator clocked at $20 \mathrm{MHz} \pm$ $600 \mathrm{~Hz}(30 \mathrm{ppm})$, allowing for a high resolution, high-frequency pulse-width modulated (PWM) square wave signal to be generated as the primary pre-amplified source for the emitted sound. In this case the duty cycle is fixed at 50\% (i.e. the output signal spends $50 \%$ of the period at a high amplitude and the other $50 \%$ low). The PWM amplitude (volume) is adjustable via a $10 \mathrm{kOhm}, 10$-turn, rotary potentiometer with stops (i.e. a dial with fine "clicks"). Frequency is modified in software and stored in program memory based on application, while the amplitude is adjusted by running the PWM output through a passive voltage-divider circuit, allowing for smooth, analogue adjustment. For applications requiring adjustable frequency, a 10-turn potentiometer can be added to provide external frequency control (resolution depending on the frequency). The divided PWM signal is then passed through a buffering amplifier (TL074, Texas Instruments, Dallas, TX) prior to stimulating an externally connected piezoelectric element (base resonant frequency at $4.4 \mathrm{kHz}$ ). The piezoelectric crystal is mounted into a custom bracket to ensure proper orientation and distance to achieve a physiologically relevant, accurate and repeatable response in the microphone (transducer) of interest. A custom bracket can be made to suit any microphone setup desired. It is important to note that the mounting bracket used to connect the transducer to the CURT was custom made to fit our transducers (the CM16/CMPA40-5V [Avisoft-Bioacoustics, Berlin, Germany]), and would need to be customized to fit other transducers. The housing holds the transducer face parallel to, and approximately $1 \mathrm{~cm}$ from, the surface of the piezoelectric element, which was secured to the base of the acrylic bracket using cyanoacrylate glue. The calibration bracket containing the piezoelectric element was connected electrically to the driving circuit through a standard $1 / 8$ " audio jack. On the exterior of the unit, a cluster of four indicators LEDS are visible to allow for basic functionality readout. Additionally, a connection for a liquid crystal display is provided for diagnostic purposes. Finally, circuitry is included for future optional stand-alone calibration 
capability—allowing the CURT to provide real-time feedback to those users without access to a computer-driven recording apparatus.

\section{Methods}

\subsection{Overview of the validation system}

To verify our calibration method/device, we made use of the Avisoft CM16/CMPA40-5V adjustable-gain microphone system (Avisoft Bioacoustics, Berlin, Germany) connected to a National Instruments DAQ (PCI-6132) and collected audio at $1 \mathrm{MS} / \mathrm{s}$ and 14-bit resolution (National Instruments, Austin, Texas). All microphones were calibrated using a 42016.8067 $\mathrm{Hz}$ signal generated using our system described above. The amplitude output from the calibration unit was fixed at $904 \pm 2 \mathrm{mV}$ peak to peak as measured using an oscilloscope (Tektronix TDS- 2024) across the leads going to the piezoelectric crystal. The amplitude was chosen to produce a sound comparable to the average magnitude of a $42 \mathrm{kHz}$ vocalization emitted by the Sprague-Dawley rat pups used in our own lab (visualized in Figure 3, with resulting amplitudes seen in Figure 6). This amplitude is specific to our own recording setup, and will need to be carefully considered by other researchers when determining the setting for their own calibration system, based on factors including transducer distance from subject, age of subject, stimuli used to elicit vocalization, etc.

One hour prior to calibration, all of the microphone and recording electronics were turned on and allowed to warm up in order to ensure a maximally stable microphone calibration response (non-linear behaviors were noted in the Avisoft equipment when not allowed to warm-up). Microphone signals were visualized using a custom written LabView (National Instruments, Austin, TX) program.

In order to assess the functionality and reliability of the calibration device, we performed four basic experiments to ensure reproducibility of data. First, an assessment was made to determine the variability amongst transducers. Second, an assessment of the thermal effects of electronics heating was conducted. Third, we assessed the reproducibility of data collected using different transducers calibrated with our system. Finally, we compared data from rat vocalization recordings between calibrated and non-calibrated systems to highlight the improvement in data quality resulting from accurate calibration. All recordings and calibrations were done in a closed environment (sound attenuating cubical), to reduce environmental noise that may alter the amplitude observed by the transducer.

\subsection{Assessment of Variability of Transducers}

To demonstrate the need to calibrate the microphones, the variability of the individual microphones was compared by choosing one microphone as a reference. The reference microphone was calibrated to $2.5 \mathrm{~V}(50 \%$ of the $5 \mathrm{~V}$, maximum output) using the CURT and the signal was monitored using a LabView program every 5 seconds. Next, each remaining microphone was exchanged for the reference microphone, and without recalibrating, the signal level was measured on the microphone. The output from each microphone was measured 30 times in order to collect an average signal level for each. Average voltage output for the four microphones was compared using Analysis of Variance, followed by Tukey posthoc tests. This comparison is used to determine variability in amplitude measurement between transducers, which have no capacity for calibration, as opposed to amplifiers, which can be calibrated. Such variability would demonstrate the need for calibration of each specific transducer/amplifier pair used for recording. 


\subsection{Assessment of Thermal Effects}

The effects of the electronic components heating were assessed using an infrared camera (FLIR i7, FLIR Systems AB, Sweden) to measure the temperature of the electronics over the course of an hour as soon as the unit was turned on. A microphone was calibrated using the CURT and voltage drift was monitored over time. Simultaneously, the temperatures of various key components of the system were monitored. This assessment allowed us to determine an appropriate length of time required for recording equipment 'warm-up' prior to use, as well assess the need for feedback circuitry in the calibration unit.

\subsection{Assessment of Data Reproducibility}

In order to ascertain the efficacy of the calibration in terms of data reproducibility, two microphones were calibrated using the CURT on their respective amplifiers and used to record a $42 \mathrm{kHz}$ signal of unknown amplitude. For each unit, 30 data points (one every 5 seconds) were recorded and compared as a measure of the accuracy of our proposed calibration method. Resulting means were compared using a two-tailed T test.

\subsection{Assessment of Comparability in Animal Vocalization Recording}

A series of ultrasonic vocalizations were recorded from a single Sprague-Dawley rat pup on postnatal day 5 (see Figure 3 for sonogram), using the recording equipment described above. This recording was then played back independently to 5 microphone/amplifiers, 3 of which had the gain set visually (analog dial placed in the same position), and 2 of which were calibrated using the CURT. Playback was accomplished using the ScanSpeak ultrasonic speaker (Avisoft Bioacoustics; Berlin, Germany) connected to a computer via a National Instruments analog output card (PCI-6731), and re-recorded using the same equipment as above. The maximum amplitude for each re-recorded vocalization was then determined using SASLab Pro (Avisoft Bioacoustics; Berlin, Germany) for comparison. This comparison will allow us to determine if variability in amplification has real-world implications on data quality, and if improvements can be made following accurate calibration with the CURT.

\section{Results}

\subsection{Assessment of Variability of Transducers}

To demonstrate the need to calibrate each transducer to each amplifier, we tested the variability in the transducers by comparing three uncalibrated transducers to one that was calibrated to $50 \%$ of its maximum output. Figure 4 shows a comparison of the four transducers. Statistical analysis showed that all three uncalibrated microphones differed significantly from each other [main effect: $\mathrm{F}(3,116)=15835.14$, $\mathrm{p}<0.001$; Mic 1 vs Mic 2: $\mathrm{p}<0.001$; Mic 1 vs Mic 3: $\mathrm{p}<0.001$; Mic 2 vs Mic 3: $\mathrm{p}<0.001]$, as well as from the calibrated microphone $(\mathrm{p}<0.001)$. Note that the output from uncalibrated microphone 1 is $3.95+0.03 \mathrm{~V}$, which corresponds to an approximate $1.2 \mathrm{~dB}$ difference from the $2.5 \mathrm{~V}$ calibrated signal. Uncalibrated microphone 3, on the other hand, shows an average uncalibrated voltage of $1.19+0.08 \mathrm{~V}$, which corresponds to an approximately $7 \mathrm{~dB}$ difference from the calibrated $2.5 \mathrm{~V}$ signal. Similarly, the variability between the uncalibrated microphones 1 and 4 corresponds to an approximately $9 \mathrm{~dB}$. Such large variations in amplitude measurement of a constant amplitude signal signify the importance of calibration.

\subsection{Thermal Effects}

We found an approximately linear relationship between time and the output voltage of the microphone prior to 50 minutes, which was likely due to temperature increases in the individual components of the microphone. These data suggest the importance of a "warm- 
up" period for both the microphones and amplifiers prior to calibration or data collection.

The CURT demonstrated no substantial need for a warm-up period.

\subsection{Assessment of Data Reproducibility}

Once microphones and amplifiers have been calibrated with the CURT, output from them is reliable, as shown in Figure 5. Variability between calibrated microphones corresponds to less than a $0.3 \mathrm{~dB}$ difference in output amplitude, and microphones show a similar variability. It is important to note that there was a small drift upwards in the signal over time, likely representative of the relationship between temperature and signal as discussed above. This thermal noise is likely the source of most variability seen in the data, but did not result in a relevant difference between the microphones' outputs.

\subsection{Assessment of Comparability in Animal Vocalization Recording}

Once microphones and amplifiers have been calibrated with the CURT, output from them is very comparable (see Figure 6). The three microphones and amplifiers that were manually adjusted to a similar level of gain showed a $10 \mathrm{~dB}$ difference from each other maximally, while the two systems calibrated using the CURT showed $1 \mathrm{~dB}$ difference maximally. The differences found in the manually adjusted microphone systems match the variability shown above in Section 4.1., further demonstrating the importance of accurate calibration, and the improvement in data quality following calibration with the CURT.

\section{Conclusions}

The CURT is a relatively simple device that allows for rapid, accurate calibration of a variety of microphones and amplifiers to ensure the reproducibility of data collected from such systems. This device is capable of emitting a constant frequency and amplitude using a microcontroller driving a piezoelectric element housed within a bracket. Prior to this system's development, manual microphone calibration did not provide consistent results, as there were too many sources of error. Visually tuning multiple amplifiers (positioning the volume knob at the same place) is unfavorable, because despite the amplifiers being set to approximately the same physical location each time, there is significant variability in intermic amplification levels and transducer representation of amplitude, likely due to manufacturing variability. Our experience has helped us determine a generic set of steps that can be applied to the calibration of any vocalization transducer:

1. Design a bracket into which the transducer can be securely and reproducibly located in front of the emitting element of the CURT. This design should be based upon the application and requirements of the user.

2. Determine the frequency and amplitude $(\mathrm{dB})$ range in which the recording transducer must operate based on the specific intended application.

3. Select the amplitude and frequency on the CURT.

4. Turn on ALL recording components and allow them to stabilize for at least 1 hour.

5. Using the output from the CURT, calibrate the transducer amplification circuitry such that the output from the recording transducer circuitry lies at exactly $50 \%$ of its peak value.

6. Repeat calibration for each microphone (note, if the microphones and amplifiers have been plugged in for at least 1 hour, no additional warm-up time is necessary, although 10 minutes is recommended if a 'cold' microphone is placed in a 'warm' pre-amplifier). 
Microphones calibrated with the CURT show dramatically reduced variability compared to visual tuning (manually adjusting the analog gain dial to the same position), allowing for the comparison of data from different occasions and across multiple microphones/amplifiers.

Our experience with development of CURT has also elucidated several sources of variability within the average microphone system. Major sources of calibration error include loose or weak cable connections and extensions, recording equipment temperature, and transducer positioning. These sources of error absolutely necessitate the careful calibration of transducers using sources of known frequency and amplitude. Furthermore, positional variations indicate the importance of secure bracket design. With the aid of a well-designed bracket and a known frequency and amplitude emitting source, reproducible and accurate calibration of transducers can be achieved. Well-calibrated transducers allow the ability to quantitatively compare data across space, animals and even experiments. The authors feel that CURT offers the design flexibility, simplicity and robustness to allow nearly any vocalization type to be accurately recorded and quantified.

\section{Supplementary Material}

Refer to Web version on PubMed Central for supplementary material.

\section{Acknowledgments}

We would like to acknowledge the assistance of Dr. Josephine Johns, Dr. Sheryl Moy, Lorinda Baker, Stephen Hampton, Thomas Jarrett, Sandy Zeskind, and Steven Emanuel. This project was funded by the following grants: F31 DA026251, P01 DA022446, P30 HD003110, and R01 MH080069.

\section{References}

1. Zeskind PS, McMurray MS, Garber KA, Neuspiel JM, Cox ET, Grewen KM, Mayes LC, Johns JM. Development of translational methods in spectral analysis of human infant crying and rat pup ultrasonic vocalizations for early neurobehavioral assessment. Front Psychiatry. 2011; 2:56. [PubMed: 22028695]

2. Nyquist H. Certain topics in telegraph transmission theory. Trans. AIEE. 1928; 47:617-644.

3. Shannon CE. Communication in the presence of noise. Proc. Institute of Radio Engineers. 1949; 37(1):10-21. 


\section{Highlights}

- $\quad$ No devices currently exist to calibrate the amplification of vocalization recordings

- $\quad$ Calibration Unit for Recording Transducers (CURT) is designed to full this need

- $\quad$ It emits a tunable frequency and amplitude in human-sonic and -ultrasonic ranges

- $\quad$ It is highly portable and fits a variety of microphone connector types

- We demonstrate the reliability of the unit and show the improvement in data quality 


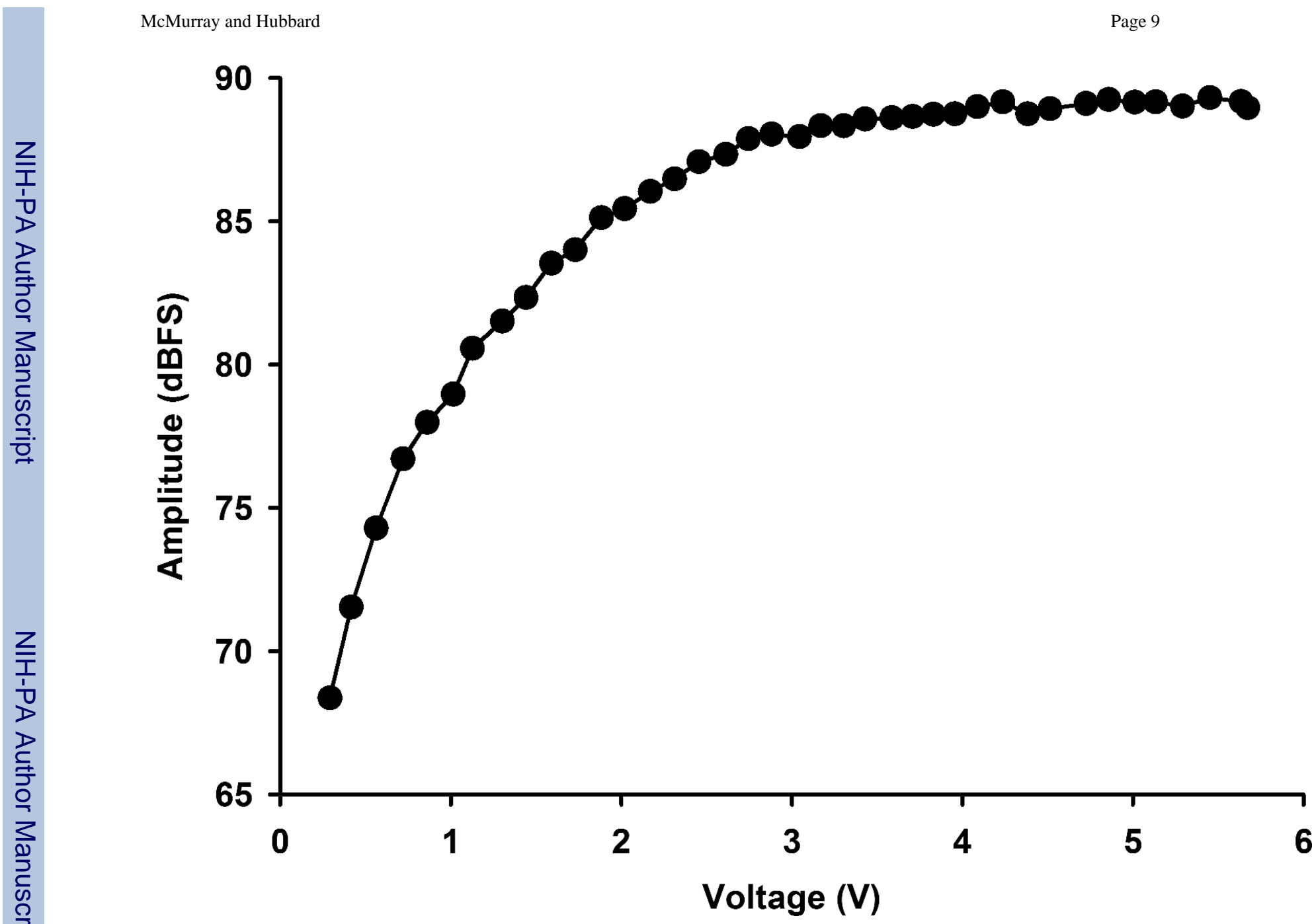

Figure 1.

The relationship between voltage and sound amplitude (relative decibels) in our recording equipment. This data is reflective of the typical exponential relationship between the two measures. Details of recording equipment are listed in text. 


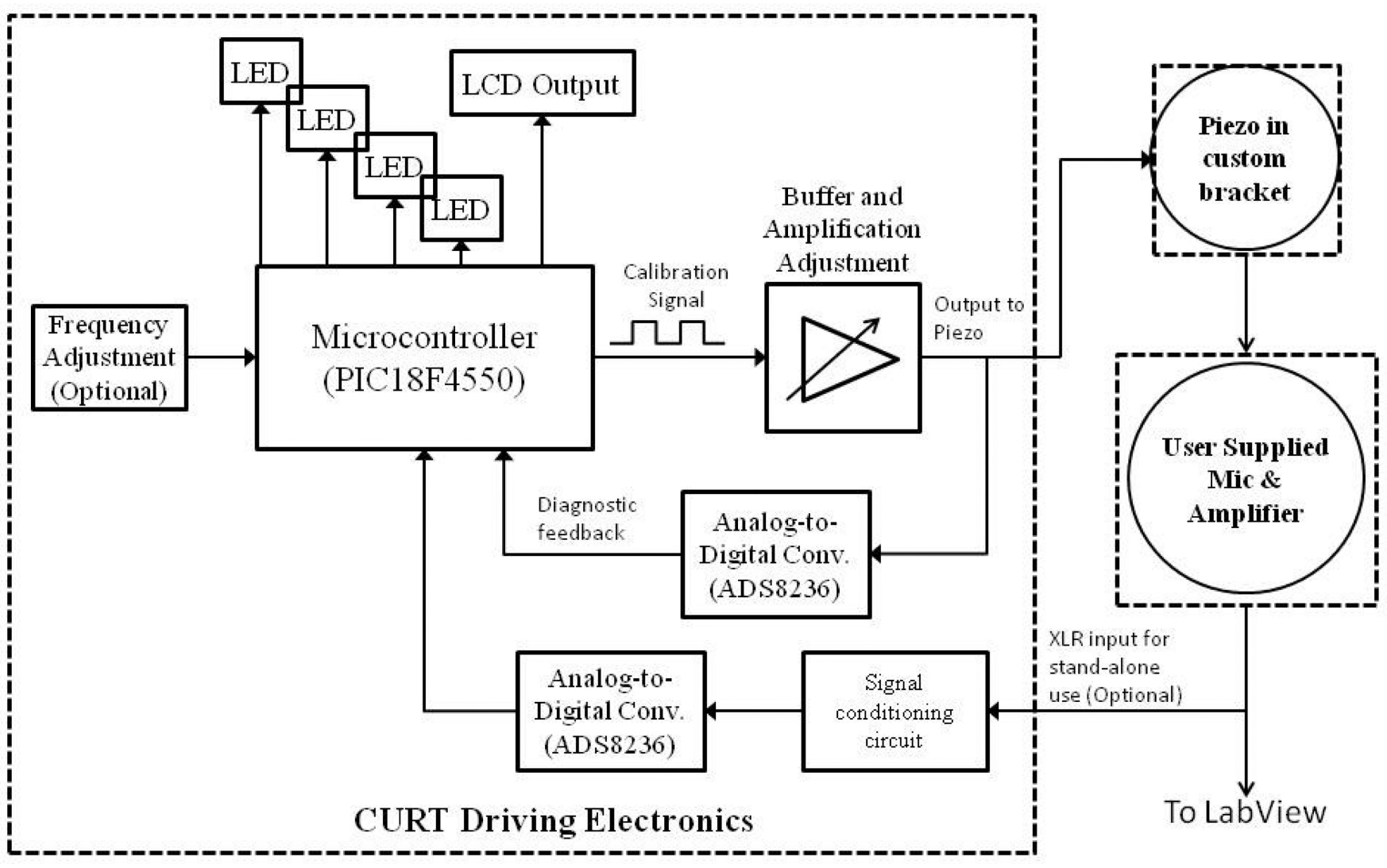

Figure 2.

Circuit flow diagram of the Calibration Unit for Recording Transducers (CURT). The main circuit (represented by the large rectangular box) generates a signal of known amplitude and frequency derived from an internal $20 \mathrm{MHz}$ crystal and amplified before being passed to an external piezo electric element that is secured in a custom mounting bracket to fit the transducer to be calibrated (supplied by the user). The system is designed to fit a variety of microphone types, emit a tuneable frequency and amplitude, and is a highly portable, standalone unit. The CURT is also very low cost to build (under 250.00 USD). 


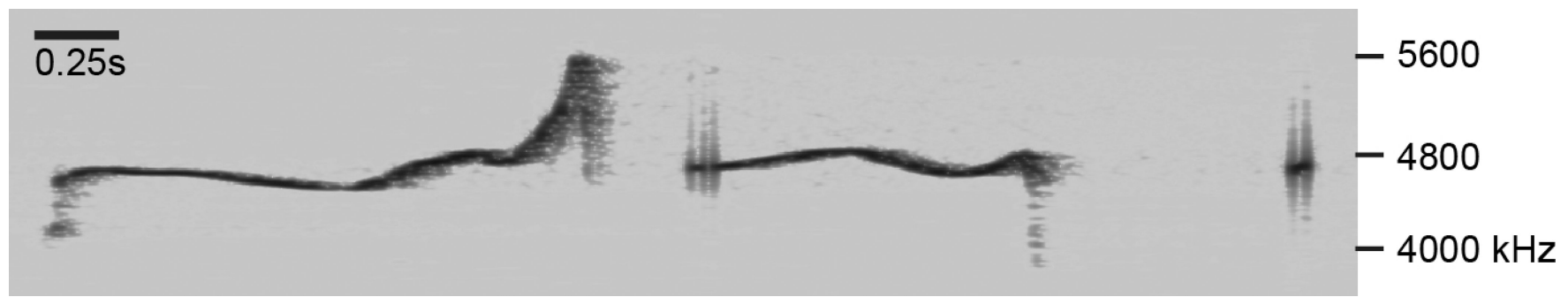

Figure 3.

Sonogram of the recorded rat pup vocalization used in the comparability study. The recording includes three vocalizations of different waveform shape, frequency range, and amplitude. 


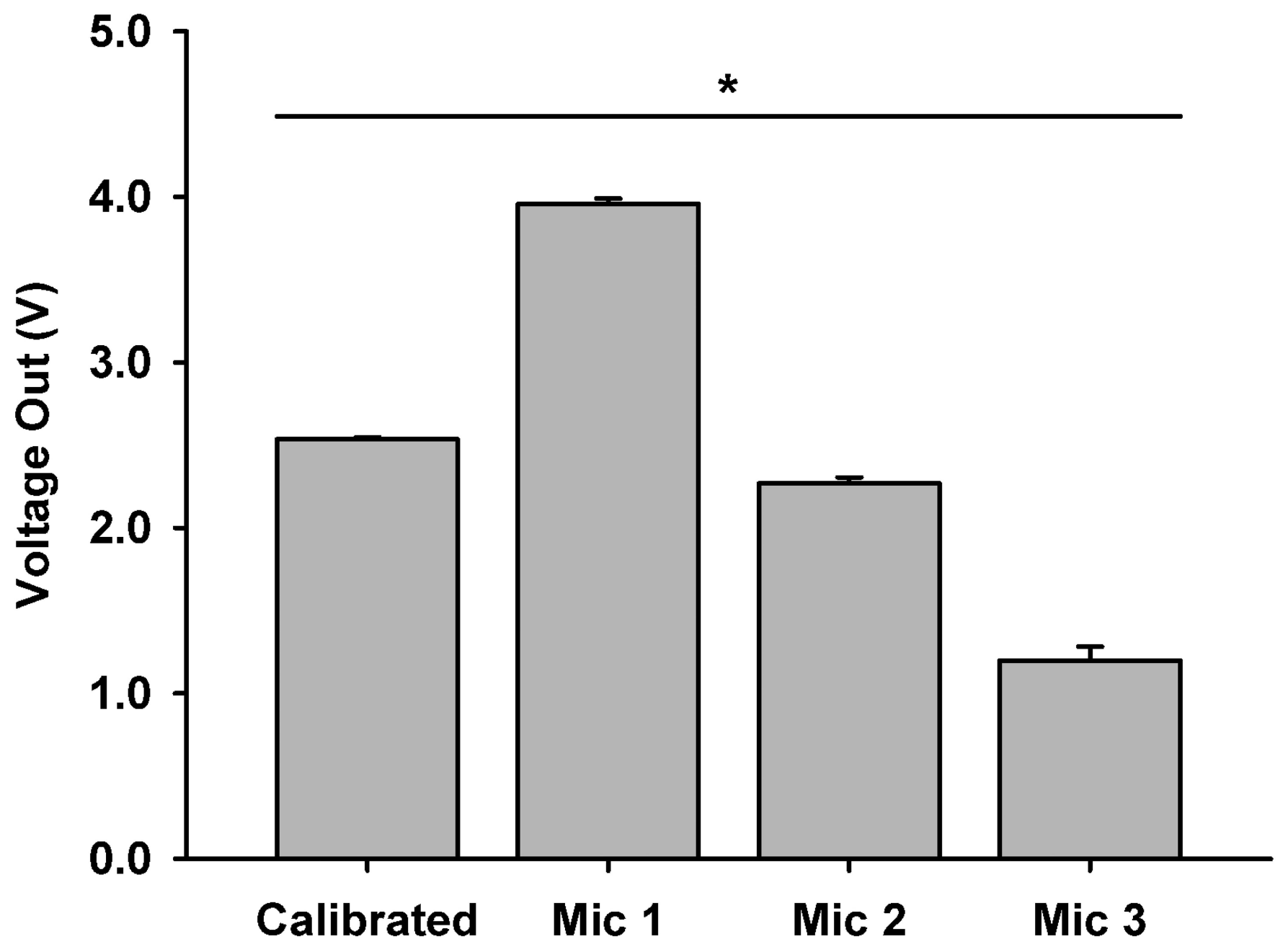

Figure 4.

Comparison of three non-calibrated transducers/amplifiers (Mic) to one calibrated with the CURT. Amplifiers of the three non-calibrated systems were set to the same approximate level of amplification via positional feedback of the dial, not the digital feedback available through the CURT. The maximal variation observed between uncalibrated systems corresponds to an approximately $9 \mathrm{~dB}$ difference in measured output, signifying the importance of digital calibration. Asterisk indicates that all uncalibrated microphones differed from each other $(\mathrm{p}<0.001)$, as well as the calibrated microphone $(\mathrm{p}<0.001)$. 


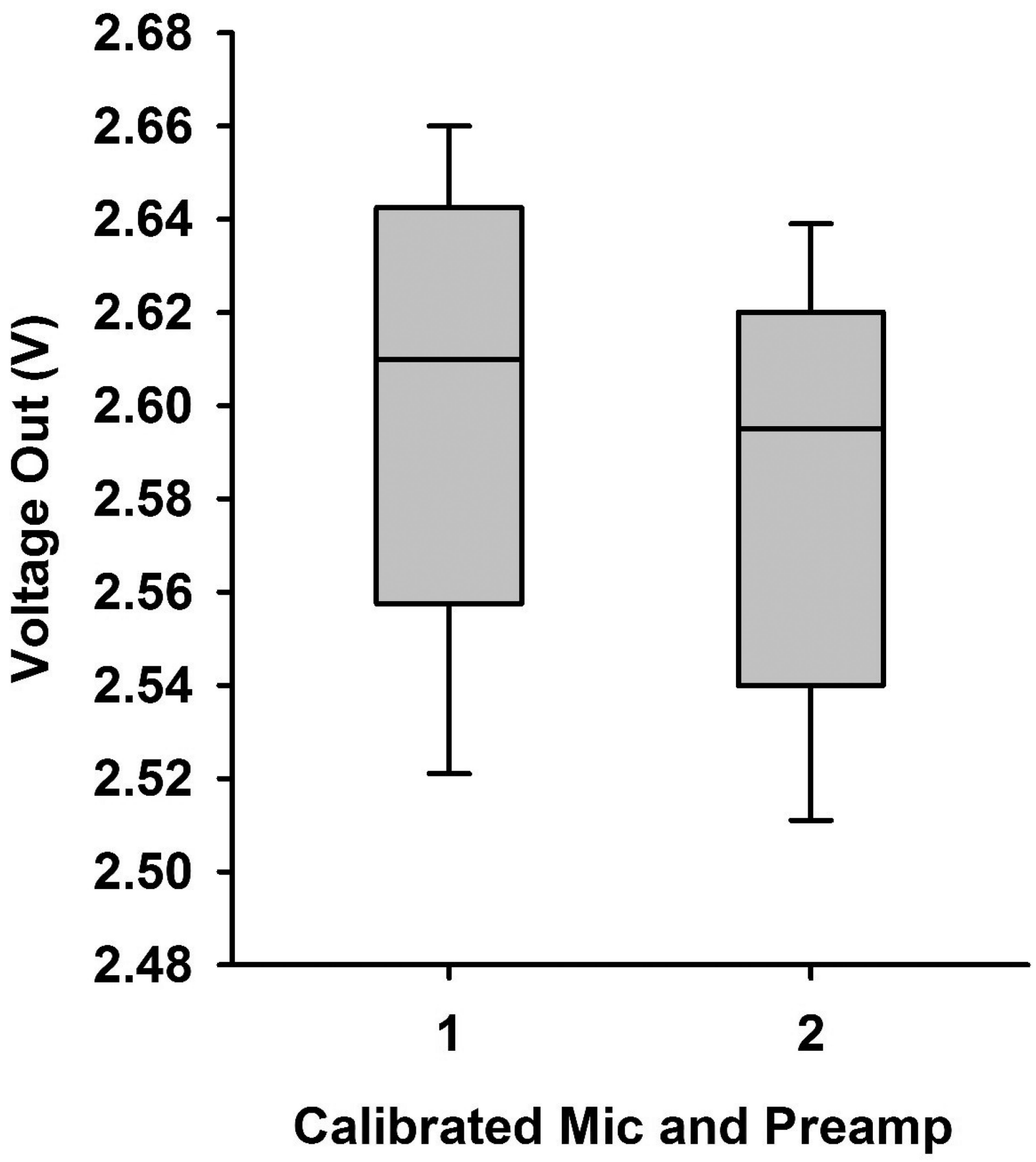

Figure 4.

Comparison of voltage output from two microphones (Mic) and preamplifiers calibrated using the CURT. Variability between microphones corresponds to less than $0.3 \mathrm{~dB}$ difference (ns) in output amplitude, and microphones show similar levels of variability. 


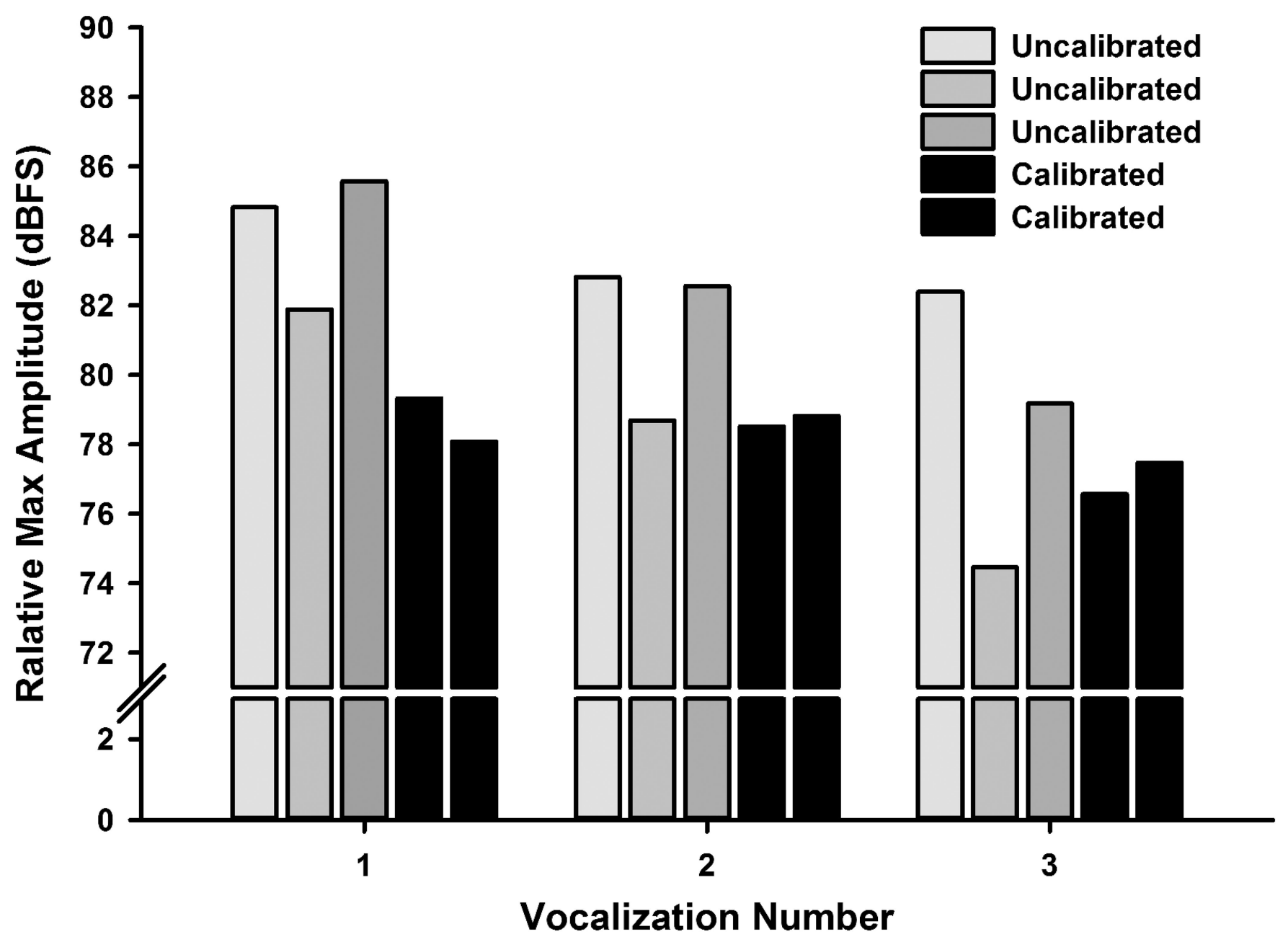

Figure 6.

Comparison of maximum amplitude of three rat pup vocalizations from five microphones/ amplifiers. The gain on three of the amplifiers was set to the same physical location on the analog dial (Uncalibrated), while the remaining two amplifiers were calibrated using the CURT system. 